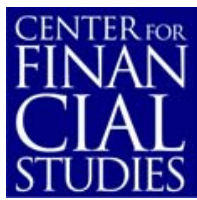

No. $2004 / 15$

\title{
Are Stationary Hyperinflation Paths \\ Learnable?
}

Klaus Adam, George W. Evans, Seppo Honkapohja 


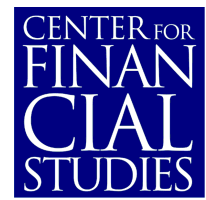

\section{Center for Financial Studies}

The Center for Financial Studies is a nonprofit research organization, supported by an association of more than 120 banks, insurance companies, industrial corporations and public institutions. Established in 1968 and closely affiliated with the University of Frankfurt, it provides a strong link between the financial community and academia.

The CFS Working Paper Series presents the result of scientific research on selected topics in the field of money, banking and finance. The authors were either participants in the Center's Research Fellow Program or members of one of the Center's Research Projects.

If you would like to know more about the Center for Financial Studies, please let us know of your interest.

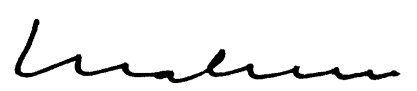

Prof. Dr. Jan Pieter Krahnen

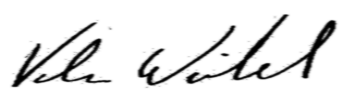

Prof. Volker Wieland, Ph.D. 
CFS Working Paper No. 2004/15

\title{
Are Stationary Hyperinflation Paths Learnable?*
}

\author{
Klaus Adam', George W. Evans ${ }^{2}$ and Seppo Honkapohja ${ }^{3}$
}

March 17, 2003

\begin{abstract}
:
Earlier studies of the seigniorage inflation model have found that the high-inflation steady state is not stable under adaptive learning. We reconsider this issue and analyze the full set of solutions for the linearized model. Our main focus is on stationary hyperinflationary paths near the high-inflation steady state. The hyperinflationary paths are stable under learning if agents can utilize contemporaneous data. However, in an economy populated by a mixture of agents, some of whom only have access to lagged data, stable inflationary paths emerge only if the proportion of agents with access to contemporaneous data is sufficiently high.
\end{abstract}

JEL Classification: C62, D83, D84, E31

Keywords: Indeterminacy, inflation, stability of equilibria, seigniorage

\footnotetext{
* Financial support from US National Science Foundation, the Academy of Finland, Yrjö Jahnsson Foundation, Bank of Finland and Nokia Group is also gratefully acknowledged.

${ }^{1}$ Klaus Adam, CEPR, London, University of Frankfurt, Mertonstr.17, PF94, 60054 Frankfurt am Main, Germany

${ }^{2}$ George W. Evans, University of Oregon, United States

${ }^{3}$ Seppo Honkapohja, Cambridge University, United Kingdom
} 


\section{Introduction}

The monetary inflation model, in which the demand for real balances depends negatively on expected inflation and the government uses seigniorage to fund in part its spending on goods, has two steady states and also perfect foresight paths that converge to the high inflation steady state. ${ }^{1}$ The paths converging towards the high steady state have occasionally been used as a model of hyperinflation, see e.g. (Fischer 1984), (Bruno 1989) and (Sargent and Wallace 1987). However, this approach remains controversial for several reasons. First, the high inflation steady state has "perverse" comparative static properties since an increase in seigniorage leads to lower steady state inflation. Second, recent studies of stability under learning of the high inflation steady state suggest that this steady state may not be a plausible equilibrium.

(Marcet and Sargent 1989) and (Evans, Honkapohja, and Marimon 2001) have shown that the high inflation steady state is unstable for various version of least squares learning. (Adam 2003) has obtained the same result for a sticky price version of the monetary inflation model with monopolistic competition. (Arifovic 1995) has examined the model under genetic algorithm learning and the economy appears always to converge to the steady state with low, rather than high inflation. Experimental work by (Marimon and Sunder 1993) also comes to the conclusion that the high inflation steady state is not a plausible outcome in the monetary inflation model.

The instability result for the high inflation steady state under learning has been derived under a particular assumption about the information sets that agents are assumed to have. (Van Zandt and Lettau 2003) raise questions about the timing and information sets in the context of learning steady states. They show that, under what is often called constant gain learning, the high inflation steady state in the Cagan model can be stable under learning with specific informational assumptions. ${ }^{2}$ In a related but different model, (Duffy 1994) showed the possibility of expectationally stable dynamic paths near an indeterminate steady state.

The monetary inflation model, like that of (Duffy 1994), has the important feature that the temporary equilibrium inflation rate in period $t$

\footnotetext{
${ }^{1}$ The model is also called the Cagan model after (Cagan 1956) .

${ }^{2}$ However, constant gain learning is most natural in nonstochastic models, since otherwise convergence to REE is precluded. In this paper we allow for intrinsic random shocks and thus use "decreasing gain" algorithms, consistent with least squares learning.
} 
depends on the private agents' one-step ahead forecasts of inflation made in two successive periods, $t-1$ and $t$. Except for some partial results in (Duffy 1994) and (Adam 2003), the different types of rational expectations equilibria (REE) in such "mixed dating models" have not been examined for stability under learning. In this paper we consider this issue, paying careful attention to different possible information sets that agents might have. In particular, we show that stationary $\mathrm{AR}(1)$ paths, as well associated sunspot equilibria around an indeterminate steady state, such as the high inflation steady state, are stable under learning when agents have access to contemporaneous data of endogenous variables. However, this result is sensitive to the information assumption. If the economy has sufficiently many agents who base their forecasts only on lagged information, then the results are changed and the equilibria just mentioned become unstable under learning.

\section{The hyperinflation model}

In the standard hyperinflation model real money demand is a linearly decreasing function of expected inflation, i.e.

$$
m_{t}^{d}=\psi_{0}-\psi_{1} E_{t}^{*} x_{t+1} \quad \text { with } \psi_{0}>\psi_{1}>0
$$

where $m_{t}^{d}$ denotes real money demand and $x_{t+1}$ the inflation factor from $t$ to $t+1$. Here $E_{t}^{*} x_{t+1}$ denotes expected inflation, which we do not restrict to be fully rational (we will reserve $E_{t} x_{t+1}$ for rational expectations). Money demand functions of the above form can be generated by monetary overlapping generations models with log-utility functions. Alternatively they can be viewed as a log-version of the (Cagan 1956) demand function.

Real money supply $m_{t}^{s}$ is given by

$$
m_{t}^{s}=\frac{m_{t-1}}{x_{t}}+g+v_{t}
$$

where $m_{t-1}$ denotes the real value of outstanding balances in $t-1, g$ is the mean value of real seigniorage, and $v_{t}$ is a stochastic seigniorage term assumed to be white noise with small bounded support and zero mean. ${ }^{3}$ This formulation of the seigniorage equation is standard, see e.g. (Sargent and

\footnotetext{
${ }^{3}$ More generally the monetary shock could be allowed to be a martingale difference sequence with small bounded support.
} 
Wallace 1987) . The above equation simply states that government purchases of goods is financed by printing money, i.e. $g+v_{t}=\left(M_{t}^{s}-M_{t-1}\right) / P_{t}$, where $M_{t}$ denotes the nominal money stock and $P_{t}$ the price of goods, so that $m_{t}=M_{t} / P_{t}$ and $x_{t}=P_{t} / P_{t-1}$. It would also be straightforward to allow for a fixed amount of government purchases financed by lump-sum taxes.

Market clearing in all periods implies that

$$
x_{t}=\frac{\psi_{0}-\psi_{1} E_{t-1}^{*} x_{t}}{\psi_{0}-\psi_{1} E_{t}^{*} x_{t+1}-g-v_{t}} .
$$

Provided

$$
g<g^{\max }=\left(\sqrt{\psi_{0}}-\sqrt{\psi_{1}}\right)^{2}
$$

there exist two noisy steady states, with different mean inflation rates $x$, given by the quadratic

$$
\psi_{1} x^{2}-\left(\psi_{1}+\psi_{0}-g\right) x+\psi_{0}=0 .
$$

We denote the low inflation steady state by $x^{l}$ and the high inflation steady state by $x^{h}$. Throughout the paper we will assume that $g<g^{\max }$ so that both steady states exist. As shown in Appendix A.1, the low inflation steady state is locally unique, while there is a continuum of stationary REE in a neighborhood of the high inflation steady state.

The model (1) can be linearized around either steady state, leading to a reduced form that fits into a general mixed dating expectations model taking the form

$$
x_{t}=\alpha+\beta_{1} E_{t}^{*} x_{t+1}+\beta_{0} E_{t-1}^{*} x_{t}+u_{t},
$$

where $u_{t}$ is a positive scalar times $v_{t}$. It is convenient to study learning within the context of the linearized model (3), and this has the advantage that our results can also be used to discuss related models with the same linearized reduced form, e.g. the one of (Duffy 1994).

The linearization of the hyperinflation model is discussed in detail in Appendix A.1. We here note that equation (1) implies $\beta_{1}>0$ and $\beta_{0}<$ 0 for the linearization at either steady state. Furthermore the coefficients $\left(\alpha, \beta_{0}, \beta_{1}\right)$ at either steady state are functions of the parameters $\omega$ and $\xi$ only, where

$$
\omega=\frac{\psi_{1}}{\psi_{0}} \quad \text { and } \quad \xi=\frac{g}{g^{\max }}
$$




\section{The mixed dating model}

We start by determining the complete set of rational expectations equilibria for model (3). These can be obtained as follows. In a rational expectations equilibrium (REE) the forecast error

$$
\eta_{t}=x_{t}-E_{t-1} x_{t}
$$

is a martingale difference sequence (MDS), which together with (3) implies that

$$
x_{t}=\alpha+\beta_{1}\left(x_{t+1}+\eta_{t+1}\right)+\beta_{0}\left(x_{t}+\eta_{t}\right)+u_{t} .
$$

Solving for $x_{t+1}$ and lagging the equation by one period delivers

$$
x_{t}=-\beta_{1}^{-1} \alpha+\beta_{1}^{-1}\left(1-\beta_{0}\right) x_{t-1}+\eta_{t}+\beta_{1}^{-1} \beta_{0} \eta_{t-1}-\beta_{1}^{-1} u_{t-1}
$$

One can decompose the arbitrary MDS $\eta_{t}$ into a component that is correlated with $u_{t}$ and an orthogonal sunspot $\eta_{t}^{\prime}$ :

$$
\eta_{t}=\gamma_{0} u_{t}+\gamma_{1} \eta_{t}^{\prime}
$$

The sunspot $\eta_{t}^{\prime}$ is again a MDS. Moreover, since $\eta_{t}$ is an arbitrary MDS, the coefficients $\gamma_{0}$ and $\gamma_{1}$ are free to take on any values. This delivers the full set of rational expectations solutions for the model:

$$
x_{t}=-\frac{\alpha}{\beta_{1}}+\frac{\left(1-\beta_{0}\right)}{\beta_{1}} x_{t-1}+\gamma_{0} u_{t}+\frac{\left(\beta_{0} \gamma_{0}-1\right)}{\beta_{1}} u_{t-1}+\gamma_{1} \eta_{t}^{\prime}+\frac{\beta_{0} \gamma_{1}}{\beta_{1}} \eta_{t-1}^{\prime}
$$

Since $\gamma_{0}$ and $\gamma_{1}$ are arbitrary there is a continuum of $\operatorname{ARMA}(1,1)$ sunspot equilibria.

For $\gamma_{0}=1$ and $\gamma_{1}=0$ we obtain the stochastic steady state solution

$$
x_{t}=\alpha\left(1-\beta_{1}-\beta_{0}\right)^{-1}+\left(1-\beta_{1}-\beta_{0}\right)^{-1} u_{t},
$$

while setting $\gamma_{0}=\beta_{0}^{-1}$ and $\gamma_{1}=0$ yields an $\operatorname{AR}(1)$ solution

$$
x_{t}=-\beta_{1}^{-1} \alpha+\beta_{1}^{-1}\left(1-\beta_{0}\right) x_{t-1}+\beta_{0}^{-1} u_{t}
$$

For the hyperinflation model, stability of steady state solutions (5) has been studied in (Marcet and Sargent 1989) ${ }^{4}$ and (Evans, Honkapohja, and

\footnotetext{
${ }^{4}$ Marcet and Sargent actually formulate the forecasting problem in terms of forecasting the price level.
} 
Marimon 2001). In this paper, we will examine the stability of the full set of $\operatorname{ARMA}(1,1)$ solutions $(4)$ and how their stability under learning is affected by the information sets. As shown in Appendix A.1, the $\operatorname{ARMA}(1,1)$ solutions near the high inflation steady state are stationary because $0<\beta_{1}^{-1}\left(1-\beta_{0}\right)<$ 1 for the linearization at $x^{h}$.

\section{Learning with full current information}

We first consider the situation where agents have information about all variables up to time $t$ and wish to learn the parameters of the rational expectations solution (4). As is well-known, the conditions for local stability under adaptive learning are given by expectational stability (E-stability) conditions. Therefore, we first discuss the E-stability conditions for the REE, after which we take up real time learning.

\subsection{E-stability}

Agents' perceived law of motion (PLM) of the state variable $x_{t}$ is given by

$$
x_{t}=a+b x_{t-1}+c u_{t-1}+d \eta_{t-1}^{\prime}+\zeta_{t}
$$

where the parameters $(a, b, c, d)$ are not known to the agent but are estimated by least-squares, and $\zeta_{t}$ represents unforecastable noise.

Substituting the expectations generated by the PLM (7) into the model (3) delivers the actual law of motion (ALM) for the state variable $x_{t}$ :

$$
\begin{aligned}
x_{t} & =\left(1-\beta_{1} b\right)^{-1}\left[\alpha+\left(\beta_{1}+\beta_{0}\right) a\right] \\
& +\left(1-\beta_{1} b\right)^{-1}\left[\beta_{0} b x_{t-1}+\left(1+\beta_{1} c\right) u_{t}+\beta_{0} c u_{t-1}+\beta_{1} d \eta_{t}^{\prime}+\beta_{0} d \eta_{t-1}^{\prime}\right]
\end{aligned}
$$

The map from the parameters in the PLM to the corresponding parameters 
in the ALM, the T-map in short, is given by

$$
\begin{aligned}
a & \rightarrow \frac{\alpha+\left(\beta_{1}+\beta_{0}\right) a}{1-\beta_{1} b} \\
b & \rightarrow \frac{\beta_{0} b}{1-\beta_{1} b} \\
c & \rightarrow \frac{\beta_{0} c}{1-\beta_{1} b} \\
d & \rightarrow \frac{\beta_{0} d}{1-\beta_{1} b}
\end{aligned}
$$

Since the variables entering the ALM also show up in the PLM, the fixed points of the T-map are rational expectations equilibria. Furthermore, as is easy to verify, all REE's are also fixed points of the T-map.

Local stability of a REE under least squares learning of the parameters in (7) is determined by the stability of the differential equation

$$
\frac{d(a, b, c, d)}{d \tau}=T(a, b, c, d)-(a, b, c, d)
$$

at the REE. This is known as the E-stability differential equation, and the connection to least squares learning is discussed more generally and at length in (Evans and Honkapohja 2001). If an REE is locally asymptotically stable under (9) then the REE is said to be "expectationally stable" or "E-stable."

Equation (9) is stable if and only if the eigenvalues of

$$
D T=\left(\begin{array}{cccc}
\frac{\beta_{1}+\beta_{0}}{1-\beta_{1} b} & \frac{-\left(\alpha+\left(\beta_{1}+\beta_{0}\right) a\right) \beta_{1}}{\left(1-\beta_{1} b\right)^{2}} & 0 & 0 \\
0 & \frac{\beta_{0}}{\left(1-\beta_{1} b\right)^{2}} & 0 & 0 \\
0 & -\frac{\beta_{1} \beta_{0} c}{\left(1-\beta_{1} b\right)^{2}} & \frac{\beta_{0}}{1-\beta_{1} b} & 0 \\
0 & -\frac{\beta_{1} \beta_{0} d}{\left(1-\beta_{1} b\right)^{2}} & 0 & \frac{\beta_{0}}{1-\beta_{1} b}
\end{array}\right)
$$

have real parts smaller than 1 at the REE. At the REE we have

$$
\begin{gathered}
a=-\beta_{1}^{-1} \alpha \\
b=\beta_{1}^{-1}\left(1-\beta_{0}\right) \\
c, d: \text { arbitrary }
\end{gathered}
$$

and the eigenvalues of $D T$ are given by:

$$
\lambda_{1}=1+\frac{\beta_{1}}{\beta_{0}} ; \lambda_{2}=\frac{1}{\beta_{0}} ; \lambda_{3}=1 ; \lambda_{4}=1
$$


The eigenvectors corresponding to the last two eigenvalues are those pointing into the direction of $c$ and $d$, respectively. As one would expect, stability in the point-wise sense cannot hold for these parameters and in contexts such as these, E-stability is defined relative to the whole class of ARMA equilibria. A class of REE is said then said to be E-stable if the dynamics under (9) converge to some member of the class from all initial points sufficiently near the class. We can then summarize the preceding analysis:

Proposition 1 If $\beta_{1}>0$ and $\beta_{0}<0$ or if $\beta_{1}<0$ and $\beta_{0}>1$ the set of $A R M A(1,1)-R E E$ is E-stable.

Figure 1 illustrates these conditions in the $\left(\beta_{0}, \beta_{1}\right)$-space. The light grey region indicates parameter values for which the ARMA equilibria are E-stable but explosive. If $\beta_{1}$ and $\beta_{0}$ lies in the black region, then the ARMA equilibria are both E-stable and stochastically stationary.

\section{FIGURE 1 HERE}

Since $\beta_{1}>0$ and $\beta_{0}<0$ for the high steady state in the hyperinflation model, Proposition 1 implies that the set of stationary $\operatorname{ARMA}(1,1)-\mathrm{REE}$ is E-stable.

We remark that Proposition 1 applies to any model with reduced form (3). In particular, in the model of (Duffy 1994) we have $-\beta_{1}=\beta_{0}>1$, and thus this proposition confirms his E-stability result for the stationary $\operatorname{AR}(1)$ solutions and, more generally, proves E-stability for stationary $\operatorname{ARMA}(1,1)$ sunspot solutions.

\subsection{Real time learning}

Next we consider real time learning of the set of ARMA equilibria (4). This section shows that stochastic approximation theory can be applied to show convergence of least squares learning when the PLM of the agents has AR(1) form and the economy can converge to the locally determinate $\operatorname{AR}(1)$ equilibrium (6). For technical reasons the stochastic approximation tools cannot be applied for the continuum of $\operatorname{ARMA}(1,1)$-REE. Therefore, real time learning of the class (4) REE will be considered in section 7 using simulations.

Assume first that agents have the PLM of AR(1) form, i.e.

$$
x_{t}=a+b x_{t-1}+\zeta_{t}
$$


The parameters $a$ and $b$ are updated using recursive least squares using data through period $t$, so that the forecasts are given by

$$
\begin{aligned}
E_{t}^{*} x_{t+1} & =a_{t}+b_{t} x_{t}, \\
E_{t-1}^{*} x_{t} & =a_{t-1}+b_{t-1} x_{t-1} .
\end{aligned}
$$

Substituting these forecasts into (3) yields the ALM

$$
x_{t}=\frac{\alpha+\beta_{0} a_{t-1}+\beta_{1} a_{t}}{1-\beta_{1} b_{t}}+\frac{\beta_{0} b_{t-1}}{1-\beta_{1} b_{t}} x_{t-1}+\frac{1}{1-\beta_{1} b_{t}} u_{t} .
$$

Parameter updating is done using recursive least squares i.e.

$$
\left(\begin{array}{c}
a_{t} \\
b_{t}
\end{array}\right)=\left(\begin{array}{c}
a_{t-1} \\
b_{t-1}
\end{array}\right)+\vartheta_{t} R_{t}^{-1}\left(x_{t-1}-a_{t-1}-b_{t-1} x_{t-2}\right)\left(\begin{array}{c}
1 \\
x_{t-2}
\end{array}\right),
$$

where $R_{t}$ is the matrix of second moments, which will be explicitly specified in the Appendix, and $\vartheta_{t}$ is the gain sequence, which is a decreasing sequence such as $t^{-1} .^{5}$ In Appendix A.2 we prove the following result:

Proposition 2 The AR(1) equilibrium of model (3) is stable under least squares learning (15) if the model parameters satisfy the E-stability conditions $\beta_{1}>0$ and $\beta_{0}<0$ or $\beta_{1}<0$ and $\beta_{0}>1$.

Since E-stability governs the stability of the $\mathrm{AR}(1)$ solution under least squares learning, the stationary $\mathrm{AR}(1)$ solutions in the hyperinflation model are learnable. The same result holds for the $\mathrm{AR}(1)$ solution in a stochastic version of the model of (Duffy 1994).

\section{$5 \quad$ Learning without observing current states}

The observability of current states, as assumed in the previous section, introduces a simultaneity between expectations and current outcomes. Technically this is reflected in $x_{t}$ appearing on both sides of the equation when substituting the PLM (7) into the model (3). To obtain the ALM one first has to solve this equation for $x_{t}$. Although this is straightforward mathematically, it is not clear what economic mechanism would ensure consistency

\footnotetext{
${ }^{5}$ See Chapter 2 of (Evans and Honkapohja 2001) for the recursive formulation of least squares estimation.
} 
between $x_{t}$ and the expectations based on $x_{t}$. Moreover, in the non-linear formulation there may even exist multiple mutually consistent price and price expectations pairs, as pointed out in (Adam 2003).

To study the role of the precise information assumption, we introduce a fraction of agents who cannot observe the current state $x_{t}$. Such agents in effect must learn to make forecasts that are consistent with current outcomes, which allows us to consider the robustness of the preceding results. Thus suppose that a share $\lambda$ of agents has information set

$$
H_{t}^{\prime}=\sigma\left(u_{t}, u_{t-1}, \ldots, \eta_{t}^{\prime}, \eta_{t-1}^{\prime}, \ldots, x_{t-1}, x_{t-2} \ldots\right)
$$

and cannot observe the current state $x_{t}$. Let the remaining agents have the "full $t$ "-information set

$$
H_{t}=\sigma\left(u_{t}, u_{t-1}, \ldots, \eta_{t}^{\prime}, \eta_{t-1}^{\prime}, \ldots, x_{t}, x_{t-1} \ldots\right)
$$

Expectations based on $H_{t}^{\prime}$ are denoted by $E_{t}^{\prime *}[\cdot]$ and expectations based on $H_{t}$ by $E_{t}^{*}[\cdot]$.

With the relevant economic expectations given by the average expectations across agents, the economic model (3) can now be written as

$$
\begin{aligned}
x_{t} & =\alpha+\beta_{1}\left((1-\lambda) E_{t}^{*}\left[x_{t+1}\right]+\lambda E_{t}^{\prime *}\left[x_{t+1}\right]\right) \\
& +\beta_{0}\left((1-\lambda) E_{t-1}^{*}\left[x_{t}\right]+\lambda E_{t-1}^{\prime *}\left[x_{t}\right]\right)+u_{t}
\end{aligned}
$$

As before, the PLM of agents with information set $H_{t}$ will be given by

$$
x_{t}=a_{2}+b_{2} x_{t-1}+c_{2} u_{t-1}+d_{2} \eta_{t-1}^{\prime}+\zeta_{t}
$$

while the PLM for agents with information set $H_{t}^{\prime}$ is given by

$$
x_{t}=a_{1}+b_{1} x_{t-1}+e_{1} u_{t}+c_{1} u_{t-1}+f_{1} \eta_{t}^{\prime}+d_{1} \eta_{t-1}^{\prime}+\zeta_{t}^{\prime} .
$$

Here $\zeta_{t}$ and $\zeta_{t}^{\prime}$ represent zero mean disturbances that are uncorrelated with all variables in the respective information sets. Since agents with information set $H_{t}^{\prime}$ do not know $x_{t}$, they must first forecast $x_{t}$ to be able to forecast $x_{t+1}$. The forecast of $x_{t}$ depends on the current shocks $u_{t}$ and $\eta_{t}^{\prime}$, which implies that these agents must estimate $e_{1}$ and $f_{1}$ to be able to forecast. 
Agents' expectations are now given by

$$
\begin{aligned}
E_{t}^{*}\left[x_{t+1}\right] & =a_{2}+b_{2} x_{t}+c_{2} u_{t}+d_{2} \eta_{t}^{\prime} \\
E_{t}^{\prime *}\left[x_{t+1}\right] & =a_{1}+b_{1} E_{t}^{\prime *}\left[x_{t}\right]+c_{1} u_{t}+d_{1} \eta_{t}^{\prime} \\
& =a_{1}+b_{1}\left[a_{1}+b_{1} x_{t-1}+e_{1} u_{t}+c_{1} u_{t-1}+f_{1} \eta_{t}^{\prime}+d_{1} \eta_{t-1}^{\prime}\right]+c_{1} u_{t}+d_{1} \eta_{t}^{\prime} \\
& =a_{1}\left(1+b_{1}\right)+b_{1}^{2} x_{t-1}+\left(b_{1} e_{1}+c_{1}\right) u_{t} \\
& +b_{1} c_{1} u_{t-1}+\left(b_{1} f_{1}+d_{1}\right) \eta_{t}^{\prime}+b_{1} d_{1} n_{t-1}^{\prime}
\end{aligned}
$$

and the implied ALM can be written as

$$
z_{t}=A+B z_{t-1}+C\left(\begin{array}{c}
u_{t} \\
\eta_{t}^{\prime}
\end{array}\right)
$$

where $z_{t}=\left(x_{t}, x_{t-1}, u_{t}, u_{t-1}, \eta_{t}^{\prime}, \eta_{t-1}^{\prime}\right)$ and where the expressions for $A, B$, and $C$ can be found in Appendix A.3.1.

It is important to note that the $\operatorname{ALM}$ is an $\operatorname{ARMA}(2,2)$ process and therefore of higher order than agents' PLM. This is due to the presence of agents with $H_{t}^{\prime}$ information. These agents use variables dated $t-2$ to forecast $x_{t}$. This feature has several important implications. First, while the T-map is given by the coefficients showing up in the $\operatorname{ARMA}(2,2)$-ALM (16), calculating the fixed points of the learning process now requires us to project the $\operatorname{ARMA}(2,2)-\operatorname{ALM}$ back onto the $\operatorname{ARMA}(1,1)$ parameter space. Second, it might appear that the resulting fixed points of the T-map would not constitute rational expectations equilibria, but rather what have been called 'restricted perceptions equilibria' (RPE). RPE have the property that agents' forecasts are optimal within the class of PLMs considered by agents, but not within a more general class of models. ${ }^{6}$

Because our agents estimate $\operatorname{ARMA}(1,1)$ models, and under the current information assumptions $\operatorname{ARMA}(1,1)$ PLMs generate $\operatorname{ARMA}(2,2)$ ALMs, there is clearly the possibility that convergence will be to an RPE that is not an REE. However, as we will show below, convergence will be to an $\operatorname{ARMA}(2,2)$ process that can be regarded as an overparameterized $\operatorname{ARMA}(1,1)$ REE. Therefore, the misspecification by agents is transitional and disappears asymptotically.

The projection of the $\operatorname{ARMA}(2,2)$-ALM on the $\operatorname{ARMA}(1,1)-P L M$ is obtained as follows. Under the assumption that $z_{t}$ is stationary equation (16)

\footnotetext{
${ }^{6}$ The issue of projecting a higher-order ALM back to a lower-order PLM first arose in (Sargent 1991). Sargent's "reduced-order" equilibrium is a particular form of RPE.
} 
implies

$$
\operatorname{vec}\left(\operatorname{var}\left(z_{t}\right)\right)=(I-B \otimes B)^{-1} \operatorname{vec}\left(\operatorname{Cvar}\left(\begin{array}{c}
u_{t} \\
\eta_{t}^{\prime}
\end{array}\right) C^{\prime}\right)
$$

Using the covariances in (17) one can express the least squares estimates as

$$
T\left(\begin{array}{c}
b_{i} \\
e_{i} \\
c_{i} \\
f_{i} \\
d_{i}
\end{array}\right)=\operatorname{var}\left(\begin{array}{c}
x_{t-1} \\
u_{t} \\
u_{t-1} \\
\eta_{t}^{\prime} \\
\eta_{t-1}^{\prime}
\end{array}\right)^{-1} \operatorname{cov}\left(\left(\begin{array}{c}
x_{t-1} \\
u_{t} \\
u_{t-1} \\
\eta_{t}^{\prime} \\
\eta_{t-1}^{\prime}
\end{array}\right), x_{t}\right)
$$

The estimate for the constant

$$
\begin{aligned}
T\left(a_{i}\right) & =\left(1-b_{i}\right) E\left(x_{t}\right) \\
& =\left(1-b_{i}\right) \frac{A_{11}}{1-\frac{1}{1 / \beta_{1}-(1-\lambda) b_{2}}\left(B_{11}+B_{12}\right)}
\end{aligned}
$$

where $A_{11}, B_{11}$, and $B_{12}$ are elements of the ALM coefficients $A$ and $B$, as given in Appendix A.3.1. This completes the projection of the $\operatorname{ARMA}(2,2)-$ ALM onto the $\operatorname{ARMA}(1,1)-P L M$.

Using Mathematica one can then show that the following parameters are fixed points of the T-map:

$$
\begin{aligned}
& \left(a_{1}, b_{1}, e_{1}, c_{1}, f_{1}, d_{1}, a_{2}, b_{2}, c_{2}, d_{2}\right) \\
& =\left(-\alpha / \beta_{1},-\rho, \gamma_{0}, \gamma_{0} \rho-1 / \beta_{1}, \gamma_{1}, \gamma_{1} \rho,-\alpha / \beta_{1},-\rho, \gamma_{0} \rho-1 / \beta_{1}, \gamma_{1} \rho\right)
\end{aligned}
$$

where

$$
\begin{aligned}
\rho & =\frac{\beta_{0}}{\beta_{1}} \\
\gamma_{0}, \gamma_{1} & : \text { arbitrary constants }
\end{aligned}
$$

Note that the PLMs of agents with information set $H_{t}$ and $H_{t}^{\prime}$ is the same (up to the coefficients showing up in front of the additional regressors of $H_{t}^{\prime}$-agents). This is not surprising since agents observe the same variables and estimate the same PLMs.

It might appear surprising that the PLM-parameters in (18) are independent of the share $\lambda$ of agents with information set $H_{t}^{\prime}$. This is because one 
might expect that the value of $\lambda$ would affect the importance the second lags in the ALM (18) and therefore influence the projection of the $\operatorname{ARMA}(2,2)$ ALM onto the PLMs. However, it can be shown that this is not true at the fixed point (18). Calculating the ALM implied by the fixed point (18) yields:

$$
\begin{aligned}
A(L)\left[1+\left(-\frac{1}{\beta_{1}}+\rho\right) L\right] x_{t} & =A(L)\left[\gamma_{0}+\left(-\frac{1}{\beta_{1}}+\gamma_{0} \rho\right) L\right] u_{t} \\
& +A(L)\left[\gamma_{1}+\gamma_{1} \rho L\right] \eta_{t}^{\prime}+A
\end{aligned}
$$

where

$$
\begin{aligned}
A(L) & =\frac{-\beta_{1} \rho-\lambda+\beta_{1} \rho \lambda}{\beta_{1} \rho(\lambda-1)-\lambda}+\frac{-\rho \lambda+\beta_{1} \rho^{2} \lambda}{\beta_{1} \rho(\lambda-1)-\lambda} L \\
A & =\frac{\alpha\left((1+\rho) \lambda-\beta_{1} \rho(-1+\lambda+\rho \lambda)\right)}{\beta_{1}\left(\beta_{1} \rho(\lambda-1)-\lambda\right)} .
\end{aligned}
$$

The ARMA(2,2)-ALM (16) has a common factor in the lag polynomials. Canceling the common factor $A(L)$ in (19) gives the $\operatorname{ARMA}(1,1)$-REE (4). From $\rho=\frac{\beta_{0}}{\beta_{1}}$ it can be seen that the resulting $\operatorname{ARMA}(1,1)$ process is precisely the $\operatorname{ARMA}(1,1) \operatorname{REE}(4)$.

To summarize the preceding argument, the ALM is a genuine $\operatorname{ARMA}(2,2)$ process during the learning transition and this is underparameterized by the agents estimating an ARMA(1,1). However, provided learning converges, this misspecification becomes asymptotically negligible. As in the case of the $\operatorname{ARMA}(1,1)-R E E$, E-stability of the $\operatorname{ARMA}(1,1)$ fixed points are determined by the eigenvalues of the matrix

$$
\frac{d T}{d\left(a_{1}, b_{1}, e_{1}, c_{1}, f_{1}, d_{1}, a_{2}, b_{2}, c_{2}, d_{2}\right)}
$$

evaluated at the fixed points.

As a first application of our setting, we consider the model of (Duffy 1994), which depends on a single parameter because $-\beta_{1}=\beta_{0}>1$. Using Mathematica to derive analytical expressions for the eigenvalues of (20), one can show that a necessary condition for E-stability is given by

$$
\lambda<\frac{\left(\beta_{1}\right)^{2}}{2\left(\beta_{1}\right)^{2}-1} .
$$

Thus, in this model the ARMA(1,1)-REE become unstable if a high enough share of agents does not observe current endogenous variables.

We now turn to our main application, i.e. the hyperinflation model. 


\section{The hyperinflation model reconsidered}

We now consider the stability of the $\operatorname{ARMA}(1,1)$-REE in the hyperinflation model when a share $\lambda$ of agents has information $H_{t}^{\prime}$ and the remaining agents have information $H_{t}$. We first examine the case of small amounts of seigniorage $\xi \rightarrow 0$, for which the expressions for the linearization coefficients and the equilibrium coefficients become particularly simple. We then present some results for the general case $\xi>0$.

\subsection{Small amounts of seigniorage}

The linearization coefficients of the hyperinflation model for $\xi \rightarrow 0$ are given by

$$
\begin{aligned}
\lim _{\xi \rightarrow 0} \alpha & =\frac{1}{\omega} \\
\lim _{\xi \rightarrow 0} \beta_{1} & =+\infty \\
\lim _{\xi \rightarrow 0} \rho & =\frac{\beta_{0}}{\beta_{1}}=-\omega
\end{aligned}
$$

From equation (18) it then follows that in the $\operatorname{ARMA}(1,1)-\mathrm{REE}$ are given by

$$
\begin{aligned}
& \left(a_{1}, b_{1}, e_{1}, c_{1}, f_{1}, d_{1}, a_{2}, b_{2}, c_{2}, d_{2}\right) \\
& =\left(0, \omega, \gamma_{0},-\gamma_{0} \omega, \gamma_{1},-\gamma_{1} \omega, 0, \omega,-\gamma_{0} \omega,-\gamma_{1} \omega\right)
\end{aligned}
$$

E-stability of the ARMA(1,1)-REE is determined by the eigenvalues of the T-map. Analytical expressions for the eigenvalues are given in Appendix A.3.2. Four of these eigenvalues are equal to zero. Two eigenvalues are equal to one. The latter correspond to the eigenvectors pointing into the direction of the arbitrary constants $\gamma_{0}$ and $\gamma_{1}$. The remaining four eigenvalues $s_{i}$ $(i=1, \ldots, 4)$ are functions of $\omega$ and $\lambda$, and we compute numerical stability results.

\section{FIGURE 2 HERE}

For $\lambda$ values lying above the line shown in Figure 2 the $\operatorname{ARMA}(1,1)$ class of REE is E-unstable. A sufficient condition for instability is $\lambda>1 / 2$ (since then $s_{1}>1$ ). 


\subsection{The intermediate and large deficit case}

Using the analytical expressions for the eigenvalues of the matrix (20) we used numerical methods to determine critical share $\lambda$ for which the $\operatorname{ARMA}(1,1)$ REE becomes E-unstable for positive values of the deficit share $\xi$. Figure 3 displays the critical $\lambda$ values for $\xi$ values of $0.2,0.5$, and 0.95 , respectively. For $\lambda$ values lying above the lines shown in these figures, the $\operatorname{ARMA}(1,1)$ class of REE is E-unstable. For $\lambda$ values below these lines the equilibria remain E-stable.

\section{FIGURE 3 HERE}

The figure suggests that $\lambda>0.5$ continues to be a sufficient condition for E-instability of the $\operatorname{ARMA}(1,1)$ REE. However, critical values for $\lambda$ appear generally to be smaller than 0.5 , with critical values significantly lower if $\omega$ is small and $\xi$ is high. Moreover, when $\omega=0$ and $\xi \rightarrow 1$, these equilibria become unstable even if an arbitrarily small share of agents does not observe the current values of $x_{t}$.

\section{Simulations}

Because formal real time learning results cannot be proved for the $\operatorname{ARMA}(1,1)$ sunspot solutions, we here present simulations of the model under learning. These indicate that the E-stability results do indeed provide the stability conditions of this class of solutions under least-squares learning. In the illustrative simulations we set $\beta_{1}=2$ and $\beta_{0}=-0.5$ and $\alpha=0$. For these reduced form parameters the values of $a$ and $b$ at the $\operatorname{ARMA}(1,1)$ REE are $a=0$ and $b=0.75$. For these reduced form parameters the $\operatorname{ARMA}(1,1)$ REE are E-stable for $\lambda=0$, see Figure 1, and convergent parameter paths are indeed obtained under recursive least squares learning. The parameter estimates for a typical simulation, shown in Figures 4 and 5, are converging toward equilibrium values of the set of $\operatorname{ARMA}(1,1)$ REE.

\section{FIGURES 4 THROUGH 7 HERE}

In Figures 6 and 7 the share of agents with information set $H_{t}^{\prime}$ is increased to $\lambda=0.5$ and the $\operatorname{ARMA}(1,1)$ sunspot equilibria become unstable under

learning. For example, $a_{t}$ and $b_{t}$ are clearly diverging from the values of the $\operatorname{ARMA}(1,1) \mathrm{REE}$. 
These simulation results illustrate on the one hand the possibility of least squares learning converging to stationary solutions near the high inflation steady state. On the other hand these results also show that stability depends sensitively on the information available to agents when their inflation forecasts are made.

\section{Conclusions}

In this paper we have studied the plausibility of stationary hyperinflation paths in the monetary inflation model by analyzing their stability under adaptive learning. The analysis has been conducted using a reduced form that has wider applicability. For the hyperinflation model, if agents can observe current endogenous variables at the time of forecasting then stationary hyperinflation paths of the $\operatorname{AR}(1)$ and $\operatorname{ARMA}(1,1)$ form, as well as associated sunspot solutions, are stable under learning. Although this suggests that these equilibria may provide a plausible explanation of hyperinflationary episodes, the finding is not robust to changes in agents' information set. In particular, if a significant share of agents cannot observe current endogenous variables when forming expectations, the stationary hyperinflation paths become unstable under learning.

\section{A Appendices: Technical Details}

\section{A.1 Linearization of hyperinflation model}

Equation (2), which specifies the steady states, can be rewritten as

$$
x^{2}-\frac{\psi_{1}+\psi_{0}-g}{\psi_{1}} x+\frac{\psi_{0}}{\psi_{1}}=0,
$$

from which it follows that the two solutions $x^{l}<x^{h}$ satisfy $x^{l} x^{h}=\psi_{0} / \psi_{1}$ and hence that

$$
x^{l}<\sqrt{\frac{\psi_{0}}{\psi_{1}}}<x^{h} .
$$


Linearizing (1) at a steady state $x$ yields $x_{t}=\alpha+\beta_{1} E_{t}^{*} x_{t+1}+\beta_{0} E_{t-1}^{*} x_{t}+u_{t}$, where

$$
\beta_{0}=-\frac{\psi_{1} x}{\psi_{0}-\psi_{1} x} \text { and } \beta_{1}=\frac{\psi_{1} x^{2}}{\psi_{0}-\psi_{1} x} .
$$

Note that $\beta_{1}>0$ and $\beta_{0}<0$. We remark that $-\beta_{0}$ is the elasticity of real money demand with respect to inflation and that $\beta_{1}=-\beta_{0} x$.

For the linearized model the $\operatorname{AR}(1)$ or $\operatorname{ARMA}(1,1)$ solutions of the form (4) are stationary if and only if the autoregressive parameter $\beta_{1}^{-1}\left(1-\beta_{0}\right)>0$ is smaller than one. Since

$$
\beta_{1}^{-1}\left(1-\beta_{0}\right)=\frac{\psi_{0}}{\psi_{1} x^{2}},
$$

it follows that the solutions (4) are stationary near the high inflation steady state $x^{h}$, but explosive near the low inflation steady state $x^{l}$.

\section{A.2 Proof of Proposition 2}

We start by defining $y_{t-1}=\left(1, x_{t-2}\right)^{\prime}$. With this notation we write the updating for the matrix of second moments as

$$
R_{t}=R_{t-1}+\vartheta_{t}\left(y_{t-1} y_{t-1}^{\prime}-R_{t-1}\right)
$$

and make a timing change $S_{t}=R_{t+1}$ in order to write recursive least squares (RLS) estimation as a stochastic recursive algorithm (SRA). In terms of $S_{t}$ we have

$$
S_{t}=S_{t-1}+\vartheta_{t}\left(\frac{\vartheta_{t+1}}{\vartheta_{t}}\right)\left(y_{t} y_{t}^{\prime}-S_{t-1}\right)
$$

and

$$
S_{t-1}=S_{t-2}+\vartheta_{t}\left(y_{t-1} y_{t-1}^{\prime}-S_{t-2}\right)
$$

for the periods $t$ and $t-1$. For updating of the estimates of the PLM parameters we have (15), which is rewritten in terms of $S_{t-1}$ as

$$
\left(\begin{array}{c}
a_{t} \\
b_{t}
\end{array}\right)=\left(\begin{array}{c}
a_{t-1} \\
b_{t-1}
\end{array}\right)+\vartheta_{t} S_{t-1}^{-1}\left(x_{t-1}-a_{t-1}-b_{t-1} x_{t-2}\right)\left(\begin{array}{c}
1 \\
x_{t-2}
\end{array}\right)
$$


and

$$
\left(\begin{array}{c}
a_{t-1} \\
b_{t-1}
\end{array}\right)=\left(\begin{array}{c}
a_{t-2} \\
b_{t-2}
\end{array}\right)+\vartheta_{t}\left(\frac{\vartheta_{t-1}}{\vartheta_{t}}\right) S_{t-2}^{-1}\left(x_{t-2}-a_{t-2}-b_{t-2} x_{t-3}\right)\left(\begin{array}{c}
1 \\
x_{t-3}
\end{array}\right)
$$

To write the entire system as a SRA we next define $\kappa_{t}=\left(a_{t}, b_{t}, a_{t-1}, b_{t-1}\right)^{\prime}$ and

$$
\phi_{t}=\left(\begin{array}{c}
\kappa_{t} \\
v e c S_{t} \\
\operatorname{vec} S_{t-1}
\end{array}\right) \text { and } X_{t}=\left(\begin{array}{c}
x_{t-1} \\
x_{t-2} \\
x_{t-3} \\
1
\end{array}\right)
$$

With this notation the equations for parameter updating are in the standard form

$$
\phi_{t}=\phi_{t-1}+\vartheta_{t} Q\left(t, \phi_{t-1,} X_{t}\right)
$$

where the function $Q\left(t, \phi_{t-1}, X_{t}\right)$ is defined by $(22),(23),(24)$ and (25). We also write (14) in terms of general functional notation as

$$
x_{t}=x_{a}\left(\phi_{t}\right)+x_{b}\left(\phi_{t}\right) x_{t-1}+x_{u}\left(\phi_{t}\right) u_{t}
$$

For the state vector $X_{t}$ we have

$$
\begin{aligned}
\left(\begin{array}{c}
x_{t-1} \\
x_{t-2} \\
x_{t-3} \\
1
\end{array}\right) & =\left(\begin{array}{cccc}
x_{b}\left(\phi_{t-1}\right) & 0 & 0 & 0 \\
1 & 0 & 0 & 0 \\
0 & 1 & 0 & 0 \\
0 & 0 & 0 & 0
\end{array}\right)\left(\begin{array}{c}
x_{t-2} \\
x_{t-3} \\
x_{t-4} \\
1
\end{array}\right) \\
& +\left(\begin{array}{cc}
x_{a}\left(\phi_{t-1}\right) & x_{u}\left(\phi_{t-1}\right) \\
0 & 0 \\
0 & 0 \\
1 & 0
\end{array}\right)\left(\begin{array}{c}
1 \\
u_{t-1}
\end{array}\right)
\end{aligned}
$$

or

$$
X_{t}=A\left(\phi_{t-1}\right) X_{t-1}+B\left(\phi_{t-1}\right) v_{t}
$$

where $v_{t}=\left(1, u_{t-1}\right)^{\prime}$. 
The system (26) and (27) is a standard form for SRAs. Chapters 6 and 7 of (Evans and Honkapohja 2001) discuss the techniques for analyzing the convergence of SRAs. The convergence points and the conditions for convergence of dynamics generated by SRAs can be analyzed in terms of an associated ordinary differential equation (ODE). The SRA dynamics converge to an equilibrium point $\phi^{*}$ when $\phi^{*}$ is locally asymptotically fixed point of the associated differential equation. We now derive the associated ODE for our model.

For a fixed value of $\phi$ the state dynamics are essentially driven by the equation

$$
x_{t-1}(\phi)=x_{a}(\phi)+x_{b}(\phi) x_{t-2}+x_{u}(\phi) u_{t-1} .
$$

Now

$$
E y_{t} y_{t}^{\prime}=\left(\begin{array}{cc}
1 & E x_{t}(\phi) \\
E x_{t}(\phi) & E x_{t}(\phi)^{2}
\end{array}\right) \equiv M(\phi) .
$$

Defining $\epsilon_{t-1}(\phi)=x_{t-1}-a-b x_{t-2}$ we compute

$$
\epsilon_{t-1}(\phi)=\left(x_{a}(\phi)-a\right)+\left(x_{b}(\phi)-b\right) x_{t-2}(\phi)+x_{u}(\phi) v_{t}
$$

so that

$$
E \epsilon_{t-1}(\phi)\left(\begin{array}{c}
1 \\
x_{t-2}(\phi)
\end{array}\right)=M(\phi)\left(\begin{array}{c}
x_{a}(\phi)-a \\
x_{b}(\phi)-b
\end{array}\right)
$$

These results yield the associated ODE as

$$
\begin{aligned}
\frac{d}{d \tau}\left(\begin{array}{c}
a \\
b
\end{array}\right) & =S^{-1} M(\phi)\left(\begin{array}{c}
x_{a}(\phi)-a \\
x_{b}(\phi)-b
\end{array}\right) \\
\frac{d S}{d \tau} & =M(\phi)-S \\
\frac{d}{d \tau}\left(\begin{array}{c}
a_{1} \\
b_{1}
\end{array}\right) & =S_{1}^{-1} M(\phi)\left(\begin{array}{c}
x_{a}(\phi)-a_{1} \\
x_{b}(\phi)-b_{1}
\end{array}\right) \\
\frac{d S_{1}}{d \tau} & =M(\phi)-S_{1},
\end{aligned}
$$

where the temporary notation of variables with/without the subscript 1 refers to the $t$ and $t-1$ dating in the system (22), (23), (24) and (25). 
A variant of the standard argument shows that stability of the ODE is controlled by the stability of the small ODE

$$
\frac{d}{d \tau}\left(\begin{array}{c}
a \\
b \\
a_{1} \\
b_{1}
\end{array}\right)=\left(\begin{array}{c}
x_{a}(\phi)-a \\
x_{b}(\phi)-b \\
x_{a}(\phi)-a_{1} \\
x_{b}(\phi)-b_{1}
\end{array}\right) .
$$

Next we linearize the small ODE at the fixed point $a=a_{1}=a^{*} \equiv-\beta_{1}^{-1} \alpha$, $b=b_{1}=b^{*} \equiv \beta_{1}^{-1}\left(1-\beta_{0}\right)$. The derivative of $(28)$ at the fixed point can be written as $D X-I$, where

$$
D X=\left(\begin{array}{cccc}
\beta_{0}^{-1} \beta_{1} & -\beta_{0}^{-1} \beta_{1} & 1 & 0 \\
0 & \beta_{0}^{-1}-1 & 0 & 1 \\
\beta_{0}^{-1} \beta_{1} & -\beta_{0}^{-1} \beta_{1} & 1 & 0 \\
0 & \beta_{0}^{-1}-1 & 0 & 1
\end{array}\right)
$$

The eigenvalues of $D X$ are clearly zero and the remaining two roots are $1+\beta_{0}^{-1} \beta_{1}$ and $\beta_{0}^{-1}$. The local stability condition for the small ODE and hence the condition for local convergence the RLS learning as given in the statement of Proposition 2.

\section{A.3 Details on the Model with a Mixture of Agents}

\section{A.3.1 The ALM when some agents do not observe current states}

The coefficients in the ALM (16) are

$$
\begin{aligned}
& A^{\prime}=\left(\varsigma\left(\alpha / \beta_{1}+(1+\rho)\left[\lambda\left(1+b_{1}\right) a_{1}+(1-\lambda) a_{2}\right]\right) \quad 0 \quad 0 \quad 00000\right) \\
& B=\left(\begin{array}{cccccc}
\varsigma B_{11} & \varsigma B_{12} & \varsigma B_{13} & \varsigma B_{14} & \varsigma B_{15} & \varsigma B_{16} \\
\varsigma & 0 & 0 & 0 & 0 & 0 \\
0 & 0 & 0 & 0 & 0 & 0 \\
0 & 0 & \varsigma & 0 & 0 & 0 \\
0 & 0 & 0 & 0 & 0 & 0 \\
0 & 0 & 0 & 0 & \varsigma & 0
\end{array}\right)
\end{aligned}
$$




$$
C=\left(\begin{array}{cc}
\varsigma\left(\lambda\left(b_{1} e_{1}+c_{1}\right)+(1-\lambda) c_{2}+1 / \beta_{1}\right) & \varsigma\left(\lambda\left(b_{1} f_{1}+d_{1}\right)+(1-\lambda) d_{2}\right) \\
0 & 0 \\
0 & 0 \\
0 & 0 \\
0 & 0 \\
0 & 0
\end{array}\right)
$$

where $\varsigma=\left(1 / \beta_{1}-(1-\lambda) b_{2}\right)^{-1}$ and

$$
\begin{aligned}
& B_{11}=\lambda b_{1}^{2}+\rho(1-\lambda) b_{2} \\
& B_{12}=\rho \lambda b_{1}^{2} \\
& B_{13}=\lambda b_{1} c_{1}+\rho\left(\lambda\left(b_{1} e_{1}+c_{1}\right)+(1-\lambda) c_{2}\right) \\
& B_{14}=\rho \lambda b_{1} c_{1} \\
& B_{15}=\lambda b_{1} d_{1}+\rho\left(\lambda\left(b_{1} f_{1}+d_{1}\right)+(1-\lambda) d_{2}\right) \\
& B_{16}=\rho \lambda b_{1} d_{1}
\end{aligned}
$$

\section{A.3.2 Eigenvalues in the small deficit case}

For the hyperinflation model with a mixture of agents, the eigenvalues of the derivative of the T-map at the ARMA(1,1)-solution near the high-inflation steady state, for small deficit values (i.e. as $\xi \rightarrow 0$ ), are given by

$$
\begin{aligned}
& s_{1}=\frac{\lambda^{2}}{(1-\lambda)^{2}} \\
& s_{2}=\frac{(1+\rho)(-1+\rho \lambda)}{\rho(-1+\lambda+\rho \lambda)} \\
& s_{3}=-\frac{2 \rho^{3}(-1+\lambda) \lambda^{2}+\rho^{5}\left(\lambda^{2}-2 \lambda^{3}\right)+\sqrt{s}}{2 \rho^{3}(-1+\lambda)^{2}\left(1+\left(-1+\rho^{2}\right) \lambda\right)} \\
& s_{4}=\frac{2 \rho^{3}(-1+\lambda) \lambda^{2}+\rho^{5}\left(\lambda^{2}-2 \lambda^{3}\right)+\sqrt{s}}{2 \rho^{3}(-1+\lambda)^{2}\left(1+\left(-1+\rho^{2}\right) \lambda\right)} \\
& s_{5}=s_{6}=1 \\
& s_{7}=s_{8}=s_{9}=s_{10}=0
\end{aligned}
$$

where

$$
s=\rho^{6} \lambda^{2}\left(4(-1+\lambda)^{2}+\rho^{4} \lambda(-4+5 \lambda)-4 \rho^{2}\left(1-3 \lambda+2 \lambda^{2}\right)\right)
$$


and $\rho=-\omega$. 


\section{References}

AdAm, K. (2003): "Learning and Equilibrium Selection in a Monetary Overlapping Generations Model with Sticky Prices," Review of Economic Studies, forthcoming.

ArIfovic, J. (1995): "Genetic Algorithms and Inflationary Economies," Journal of Monetary Economics, 36, 219-243.

Barnett, W., J. Geweke, And K. Shell (eds.) (1989): Economic Complexity: Chaos, Sunspots, Bubbles, and Nonlinearity. Cambridge University Press, Cambridge.

Bruno, M. (1989): "Econometrics and the Design of Economic Reform," Econometrica, 57, 275-306.

Cagan, P. (1956): "The Monetary Dynamics of Hyper-Inflation," in (Friedman 1956).

Duffy, J. (1994): "On Learning and the Nonuniqueness of Equilibrium in an Overlapping Generations Model with Fiat Money," Journal of Economic Theory, 64, 541-553.

Evans, G. W., And S. HonkapohjA (2001): Learning and Expectations in Macroeconomics. Princeton University Press, Princeton, New Jersey.

Evans, G. W., S. Honkapohja, and R. Marimon (2001): "Convergence in Monetary Inflation Models with Heterogeneous Learning Rules," Macroeconomic Dynamics, 5, 1-31.

FISCHER, S. (1984): "The Economy of Israel," Journal of Monetary Economics, Supplement, 20, 7-52.

Friedman, M. (ed.) (1956): Studies in the Quantity Theory of Money. University of Chicago Press, Chicago.

Marcet, A., and T. J. Sargent (1989): "Convergence of Least Squares Learning and the Dynamic of Hyperinflation," in (Barnett, Geweke, and Shell 1989), pp. 119-137. 
Marimon, R., AND S. Sunder (1993): "Indeterminacy of Equilibria in a Hyperinflationary World: Experimental Evidence," Econometrica, 61, 1073-1107.

Razin, A., And E. SadkA (eds.) (1987): Economic Policy in Theory and Practice. Macmillan, London.

SArgent, T. J. (1991): "Equilibrium with Signal Extraction from Endogenous Variables," Journal of Economic Dynamics and Control, 15, 245-273.

Sargent, T. J., And N. Wallace (1987): "Inflation and the Government Budget Constraint," in (Razin and Sadka 1987).

VAn Zandt, T., and M. Lettau (2003): "Robustness of Adaptive Expectations as an Equilibrium Selection Device," Macroeconomic Dynamics, 7, 89-118. 


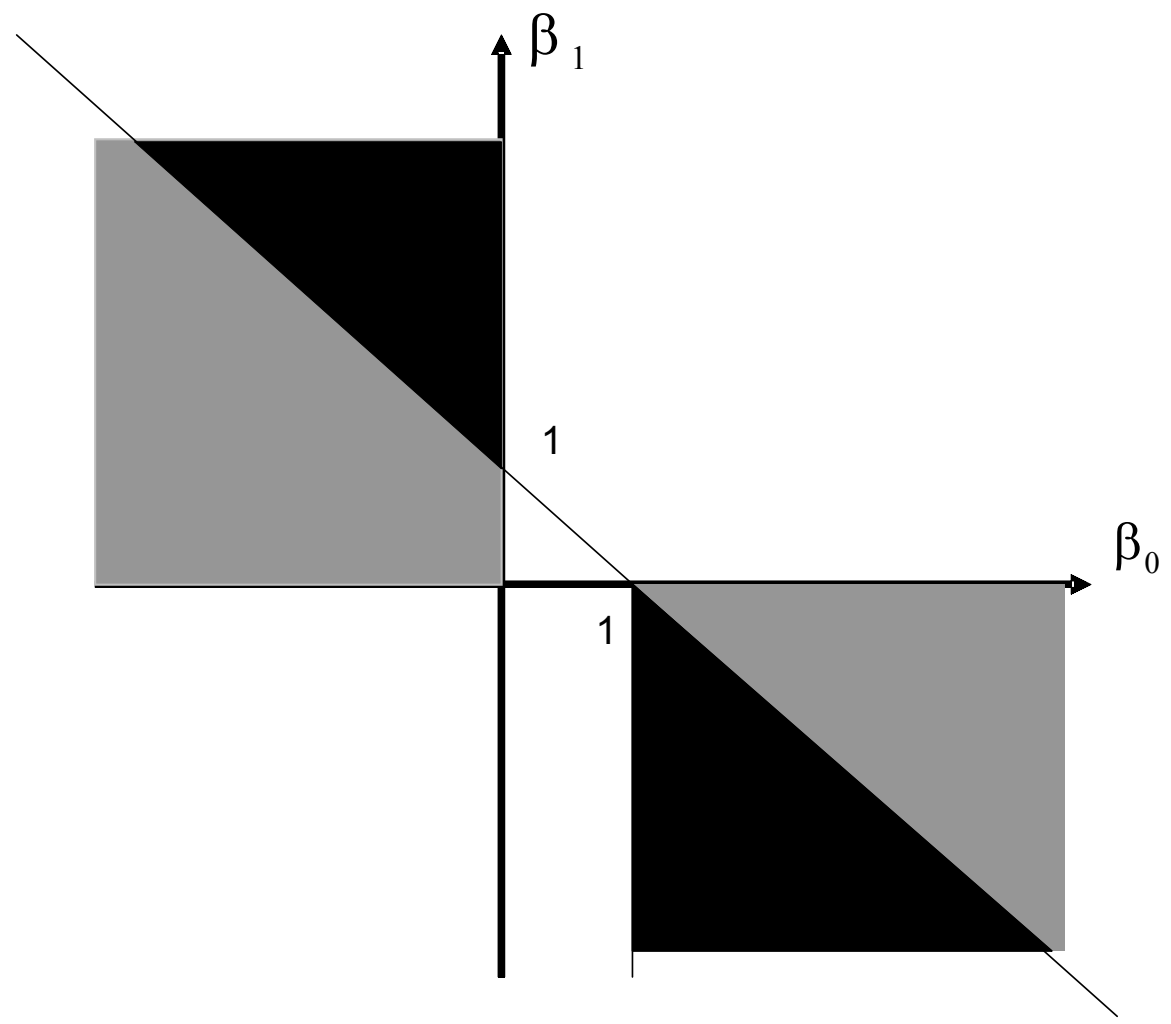

Figure 1: Regions of E-Stable ARMA equilibria 


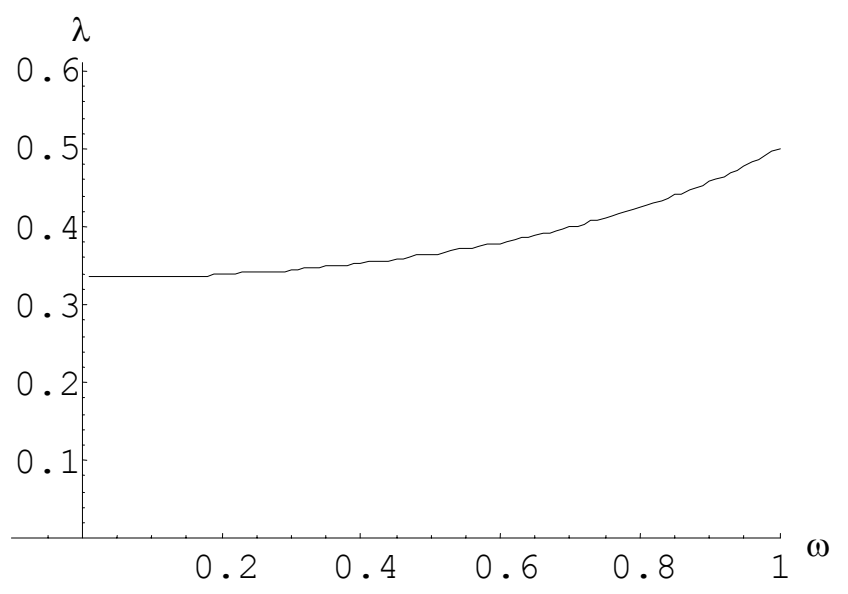

Figure 2: Critical value of $\lambda$, small deficit case $(\xi \rightarrow 0)$

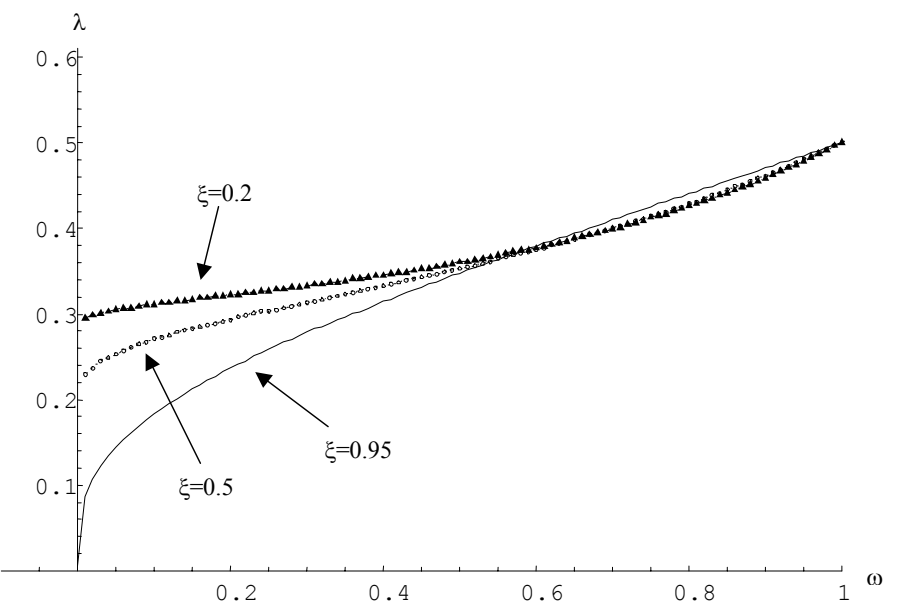

Figure 3: Critical value of $\lambda$, intermediate and large deficit case 


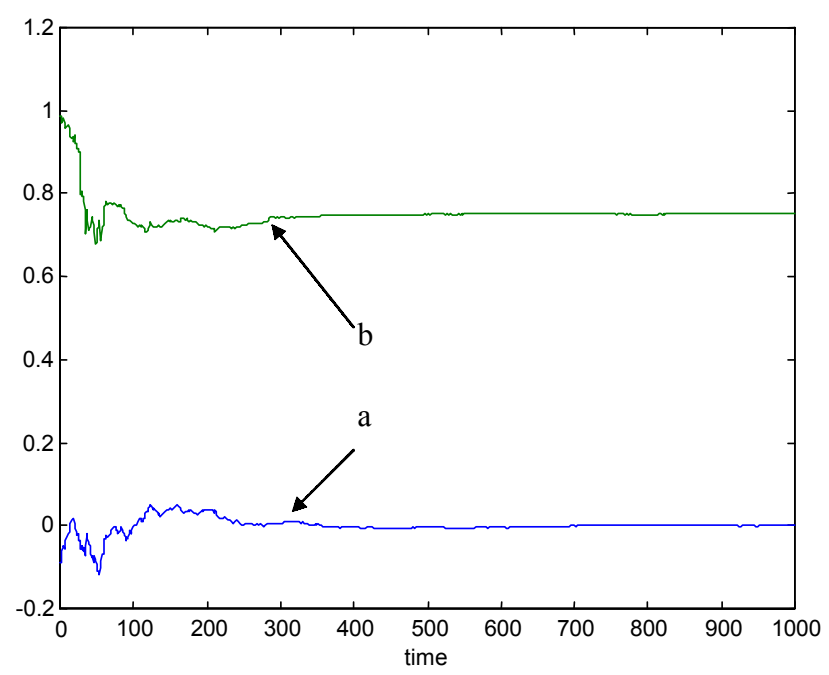

Figure 4: Example of convergence when $\lambda=0$

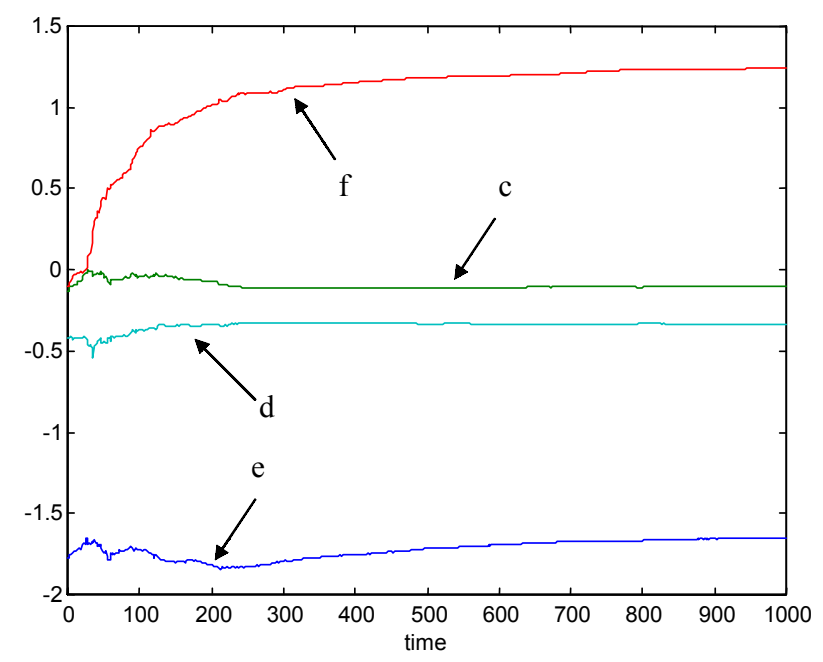

Figure 5: Example of convergence when $\lambda=0$ 


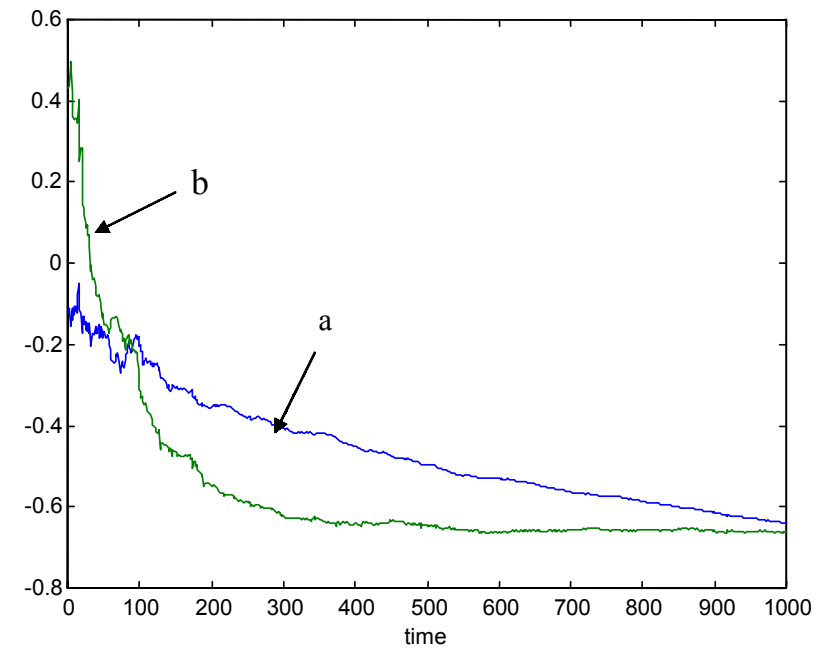

Figure 6: Example of divergence when $\lambda=0.5$

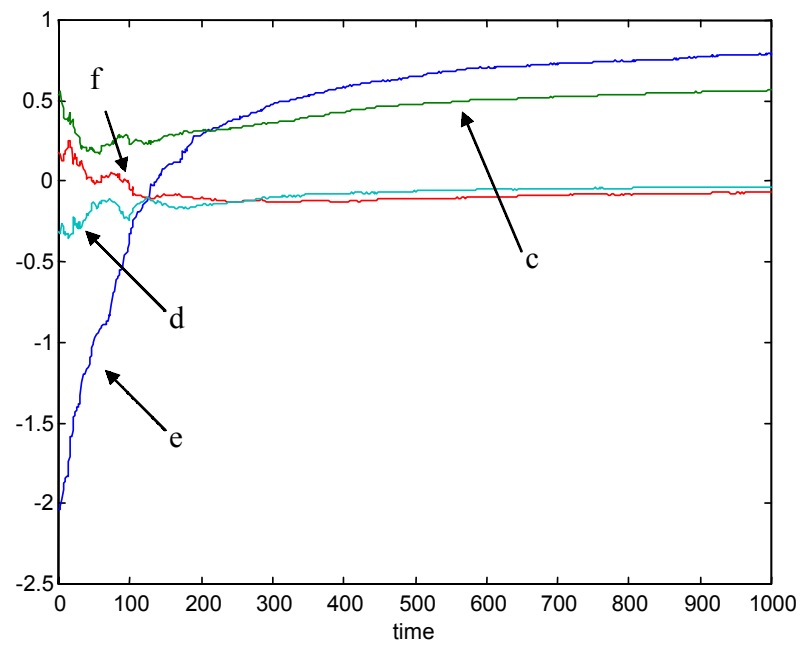

Figure 7: Example of divergence when $\lambda=0.5$ 


\section{CFS Working Paper Series:}

\begin{tabular}{lll} 
No. & Author(s) & Title \\
\hline $2004 / 05$ & $\begin{array}{l}\text { Uwe Wals } \\
\text { Douglas Cumming }\end{array}$ & $\begin{array}{l}\text { Private Equity Returns and Disclosure around } \\
\text { World }\end{array}$ \\
$2004 / 06$ & $\begin{array}{l}\text { Dorothea Schäfer } \\
\text { Axel Werwatz } \\
\text { Volker Zimmermann }\end{array}$ & $\begin{array}{l}\text { The Determinants of Debt and (Private-) Equincing in Young Innovative SMEs: Evid } \\
\text { from Germany }\end{array}$ \\
& $\begin{array}{l}\text { Michael W. Brandt } \\
\text { Francis X. Diebold }\end{array}$ & $\begin{array}{l}\text { A No-Arbitrage Approach to Range-Based } \\
\text { Estimation of Return Covariances and } \\
\text { Correlations }\end{array}$ \\
& &
\end{tabular}

2004/08 Peter F. Christoffersen

Financial Asset Returns, Direction-of-Change Francis X. Diebold

Forecasting, and Volatility Dynamics

2004/09 Francis X. Diebold Canlin Li

Forecasting the Term Structure of Government Bond Yields

2004/10 Sean D. Campbell

Weather Forecasting for Weather Derivatives

Francis X. Diebold

2004/11 Francis X. Diebold

The Nobel Memorial Prize for Robert F. Engle

2004/12 Daniel Schmidt

Private equity-, stock- and mixed asset-portfolios: A bootstrap approach to determine performance characteristics, diversification benefits and optimal portfolio allocations

$\begin{array}{ll}\text { 2004/13 } & \begin{array}{l}\text { Klaus Adam } \\ \text { Roberto M. Billi }\end{array}\end{array}$

2004/14 Günter Coenen Volker Wieland

2004/15 Klaus Adam George W. Evans Seppo Honkapohja
Optimal Monetary Policy under Commitment with a Zero Bound on Nominal Interest Rates

Exchange-Rate Policy and the Zero Bound on Nominal Interest

Are Stationary Hyperinflation Paths Learnable? 